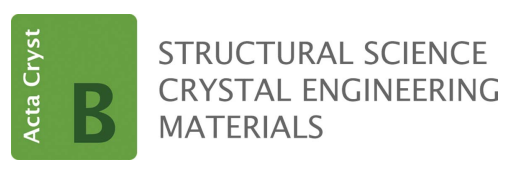

ISSN 2052-5206

Received 9 July 2019

Accepted 26 September 2019

Edited by J. Lipkowski, Polish Academy of Sciences, Poland

Keywords: extreme conditions; diamond-anvil cell; thermodynamic transformations; chemical reactions.
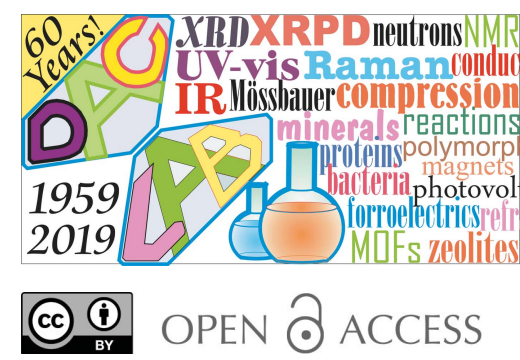

\section{Lab in a DAC - high-pressure crystal chemistry in a diamond-anvil cell}

\author{
Andrzej Katrusiak* \\ Faculty of Chemistry, Adam Mickiewicz University, ul. Uniwersytetu Poznańskiego 8, Poznań, 61-614, Poland. \\ *Correspondence e-mail: katran@amu.edu.pl
}

The diamond-anvil cell (DAC) was invented 60 years ago, ushering in a new era for material sciences, extending research into the dimension of pressure. Most structural determinations and chemical research have been conducted at ambient pressure, i.e. the atmospheric pressure on Earth. However, modern experimental techniques are capable of generating pressure and temperature higher than those at the centre of Earth. Such extreme conditions can be used for obtaining unprecedented chemical compounds, but, most importantly, all fundamental phenomena can be viewed and understood from a broader perspective. This knowledge, in turn, is necessary for designing new generations of materials and applications, for example in the pharmaceutical industry or for obtaining super-hard materials. The high-pressure chambers in the DAC are already used for a considerable variety of experiments, such as chemical reactions, crystallizations, measurements of electric, dielectric and magnetic properties, transformations of biological materials as well as experiments on living tissue. Undoubtedly, more applications involving elevated pressure will follow. High-pressure methods become increasingly attractive, because they can reduce the sample volume and compress the intermolecular contacts to values unattainable by other methods, many times stronger than at low temperature. The compressed materials reveal new information about intermolecular interactions and new phases of single- and multi-component compounds can be obtained. At the same time, high-pressure techniques, and particularly those of X-ray diffraction using the DAC, have been considerably improved and many innovative developments implemented. Increasingly more equipment of inhouse laboratories, as well as the instrumentation of beamlines at synchrotrons and thermal neutron sources are dedicated to high-pressure research.

\section{Introduction}

Now, in the first decades of the 21st century, it is clear that high-pressure research is opening a new chapter in pure sciences and technologies. For centuries, researchers realized the enormous potential of pressure effects; however, the means to perform adequate experiments were limited. For technical reasons, pressure remained neglected in many fields of science. While temperature and composition could be easily and naturally changed for the purposes of research and practical applications, the pressure equipment was very expensive, complicated and often inefficient. Presently, innovative high-pressure devices naturally add a new thermodynamic dimension to all sciences related to materials, physics, chemistry and biology. In 1959, exactly 60 years ago, the diamond-anvil cell (DAC) was constructed independently by Jamieson et al. (1959) at the University of Chicago and by Weir et al. (1959) at the National Bureau of Standards. Soon after, the DAC was equipped with a gasket enabling the generation of hydrostatic pressure (Van Valkenburg, 1962). This ingenious, simple device revolutionized high-pressure research, 
particularly in the fields of geology, astrophysics, physics and materials chemistry. This was a quiet revolution, proceeding slowly through a gradual perfection of the DAC designs and operation, as well as development of new techniques and spectacular benchmark experiments. One of the most frequently indicated capabilities of the DAC is the recordbreaking pressure. The DAC greatly increased the attainable static pressure and presently a pressure of more than $500 \mathrm{GPa}$ is claimed (Sakai et al., 2018; Dubrovinskaia et al., 2016). Even higher pressure of terapascals (TPa) has been reached by shock compression (Smith et al., 2014; Wang, Coppari et al., 2016). To place this achievement in the scale of pressure in nature, the highest pressure generated in the laboratory is higher than that at the centre of Earth (estimated as $364 \mathrm{GPa}$ ), but still lower than that in the centre of larger planets in the solar system. We still do not know the properties of matter under the pressure inside the Sun, which is often described as a micro-star, in reference to usually much larger stars in the universe. Is everything metallic and superconducting at such enormous pressure? The pressure scale extends even further and presently the maximum pressure in the universe is associated with neutron stars and black holes, where atoms collapse into the mass of neutrons. And surely this is not the end of the pressure scale! Huge pressure can occur when stellar objects collide head-on at a speed approaching that of the speed of light and, at the other end of our size scale, in hardly explored subatomic particles, for example, between gluons and quarks.

The number of high-pressure devices available in laboratories and large facilities has steadily increased and various types of high-pressure equipment - hydraulic press, belt, piston-and-cylinder, opposed anvils, DAC or laser-generated shock waves - are constantly being adapted to new types of experiments and new applications. This progress in experi-

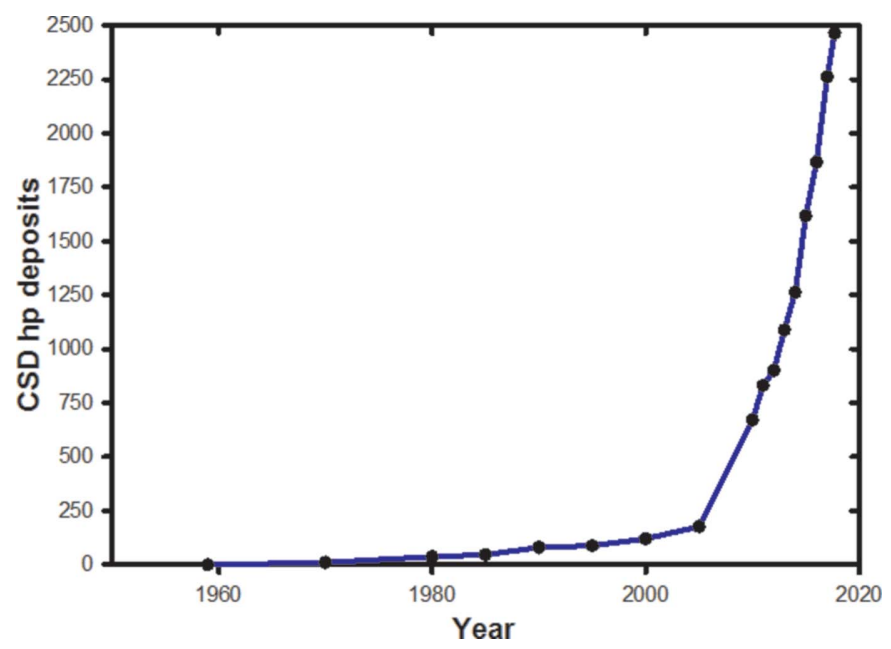

Figure 1

The number of high-pressure (hp) deposits accumulated in the Cambridge Structural Database (CSD) (Groom et al., 2016) starting from 1950, when Lawson \& Tang applied a diamond piston for generating high pressure, up to the latest release of the CSD in 2019. The analogous search in the Inorganic Crystal Structure Database (ICSD) yields twice as many, 5112 high-pressure (larger than $100 \mathrm{MPa}$ ) deposits. mental equipment is matched by the development of adequate theoretical methods for predicting high-pressure phases (Price, 2018; Oganov, 2018; Oganov et al., 2011; Grochala et al., 2007; Neumann et al., 2015; Zurek \& Bi, 2019). The increasing application of high pressure in various fields of research is illustrated in Fig. 1 by the number of crystal structures determined in high-pressure conditions and deposited in the Cambridge Structural Database (CSD) (Groom et al., 2016). Although some structural high-pressure experiments were performed before the advent of the DAC, they are few in number and the results were inaccurate. For organic compounds the first structures of the samples compressed in the DAC were reported for tetrachloromethane and phase II of benzene (Fourme, 1968; Piermarini et al., 1969).

It should be noted that progress in high-pressure experimental and theoretical methods would not be possible without the achievements in other fields of science and technology. For example, during these six decades X-ray diffractometry has become much more efficient owing to the very powerful X-ray sources, both in laboratories and in synchrotrons, multilayermirrors focusing of the beam, microbeams and highly sensitive area detectors. Computing power has increased by several orders of magnitude. The diamond anvils, initially made of brilliant-cut diamonds, the same as for jewellery, have been redesigned especially for use in DACs. Some of these parallel developments important for high-pressure research are shown in Fig. 2.

Until about 2005, high-pressure structural deposits in the CSD were sporadic, but since then the number of deposited structures has increased rapidly owing to the technological progress. This rise reflects the common use of area detectors in X-ray diffractometers, which enables data collections for the sample crystal(s) without a prior determination of the orientation matrix. Thus, the diffraction images of the sample enclosed in the DAC can be recorded and they can be analysed after concluding the data collection. Even several

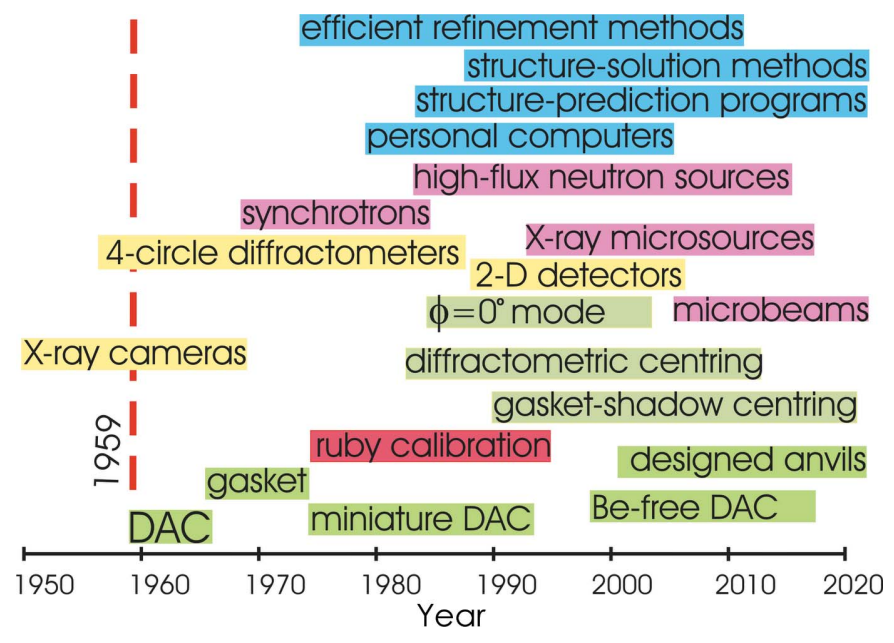

Figure 2

Calendar of selected technological developments relevant to highpressure research: in the DAC design, diffractometry, radiation sources, computational methods and related areas. The vertical dashed line marks the inception of the DAC. 
diffraction patterns from several crystal grains can be identified, separated and used for single-crystal refinements. Alternatively, diffraction patterns of several differently oriented grains of the same compound can be merged in order to improve the completeness of the diffraction data.

One of the main advances facilitating the use of the DAC was the invention of several methods for pressure calibration (see references in Jayaraman, 1983; Katrusiak, 2008, 2018). The most successful of them was the ruby luminescence scale their inventors were fully aware of the inaccurate pressure assessments in the DAC (Piermarini et al., 1969). The DAC is constantly being developed and adapted to various applications. The transparent diamond anvils are ideal for visual observations of the sample inside the DAC, and the transformations of the sample can be determined; the same or other phases can be obtained by the crystallization of melts or solutions (Van Valkenburg, 1962; Allan et al., 1998; Fabbiani \& Pulham, 2006; Allan \& Clark, 1999; Bujak et al., 2008). The sample in the DAC can be processed at the extreme conditions of very high pressure and temperature: the confined volume of the high-pressure chamber prevents the evaporation of the chosen solvents and of the sample itself. At the same time, the sample is effectively protected from contact with the atmosphere. Thus, the sample sealed in the DAC can be compressed and heated to the chosen starting pressure and temperature and then processed by changing their values. In these changing conditions one can investigate the stability regions of a compound and its phase transitions, and this information can be used for drawing the pressure-temperature $(p-T)$ phase diagrams (Katrusiak et al., 2011; Podsiadło et al., 2017), in a similar way as done by high-pressure thermal analysis and calorimetry (Barrio et al., 2012, 2017; Toscani et al., 2016). There are compounds that crystallize into new phases only after recrystallization from the melt or solution, and these phases cannot be obtained by compressing the ambient-pressure crystal. Such new phases have been termed hidden phases and they were observed e.g. for imidazole and di-p-tolyl disulfide (Paliwoda et al., 2012; Sobczak \& Katrusiak, 2017). The extreme conditions in the DAC can be applied for performing chemical reactions which otherwise would require catalysts, would proceed with much smaller yields or would not proceed at all (Sobczak et al., 2018; Półrolniczak et al., 2018; Olejniczak \& Katrusiak, 2008; Neumair et al., 2010; Huppertz, 2011).

Presently, one article cannot cover the whole variety of high-pressure crystallographic experiments, the new designs of high-pressure equipment, computer programs for theoretical computations for high-pressure structures and for the interpretation of high-pressure data. Therefore, this perspective article focuses on selected aspects most relevant to crystal engineering and the research results changing the understanding of concepts in materials chemistry, undoubtedly biased by this author's personal interests. References to highpressure effects can be found in the scientific literature on various subjects, ranging from pure sciences - such as thermodynamics, physics, chemistry and biology - to applied research and technologies - for example geology, astrophysics, super-hard materials, chemical processes, pharmacy (Coudert, 2015; Bezzu et al., 2019; Meyer et al., 2019; Zakharov et al., 2015; Moggach et al., 2008; Fabbiani \& Pulham, 2006; Lee et al., 2014; McKellar \& Moggach, 2015).

\section{DAC - a versatile apparatus}

It is well known that substances, when exposed to pressure, can change their preference to crystallize as a single- or multicomponent compound. Such a changeable preference under pressure was observed for methane-hydrogen, neon-helium, thiourea-water and other mixtures (Somayazulu et al., 1996; Loubeyre et al., 1993; Zieliński \& Katrusiak, 2015; Tomkowiak et al., 2013; Guńka et al., 2015). Remarkably, some of these solvates, requiring high pressure to be formed, do not decompose at ambient conditions (Zieliński \& Katrusiak, 2015).

The wide analytical methodology devised for the in situ characterization of compounds compressed in the DAC includes X-ray and neutron diffraction on single crystals, powders and amorphous phases, inelastic scattering, electric conductivity, elasticity, rheology (Mao et al., 1998), magnetism (Graf et al., 2011; Jackson et al., 2003; Marizy et al., 2017; Goncharenko, 2004; Pinkowicz et al., 2015), UV-visible, IR, Raman, X-ray absorption, Mössbauer and NMR (Bertani et al., 1992) spectroscopies, refractometry, micro-X-ray beam tomography and others. New techniques for in situ studies in the DAC are regularly being developed. Indeed, the DAC can be regarded as a versatile multi-purpose apparatus adaptable for various types of experiments, some of which do not require sophisticated auxiliary equipment.

\section{Towards universal laws and rules of high-pressure transformations}

The transformations induced by temperature and pressure can often be intuitively predicted. This, of course, requires a basic understanding of how the temperature and pressure interact with the material under study. Generally, one can distinguish several groups of transformations, like physical changes and chemical reactions, or reversible and irreversible deformations.

One of the most basic principles of thermodynamics states that an object reduces its volume when the pressure increases $(\partial V / \partial p<0)$. In other words, the volume compressibility $\left(\beta_{V}=\right.$ $\left.-1 / V \times \partial V /\left.\partial p\right|_{T=\text { const }}\right)$ must be positive. This fundamental law applies to closed systems, i.e. objects which do not exchange their contents with their environment. However, there are systems, like porous zeolites or metal-organic frameworks (MOFs), where the elevated pressure triggers the transport of the surrounding medium into the pores, which results in the counterintuitive effect of negative volume compressibility $\beta_{V}$ (Lee et al., 2002; Coasne et al., 2011). It means that the crystal increases its volume when pressure increases; however this effect is connected to the increased contents of pores. The negative $\beta_{V}$ is due to the exchange of the guest molecules between the environment and crystal, each of which is an open 
system. Both the liquid medium and the sample crystal (the whole contents of the DAC chamber) constitute the closed system. The transport of guests into the pores requires that the molecules of the pressure-transmitting medium can penetrate the pores. Indeed the observations of negative $\beta_{V}$ were made for penetrable liquids (Lee et al., 2002; Coasne et al., 2011). However, a similar behaviour was later also observed for nonpenetrable fluids, which separated the environment and the crystal into two closed systems (Sobczak \& Katrusiak, 2018). The negative $\beta_{V}$ has been explained by the zone-collapse effect, when a part of the sample collapses and becomes amorphous under the high pressure and the contents of pores in this collapsed region is extruded into the portion of the sample that remains crystalline. Thus the sample is differentiated into two open systems and the guest molecules are moved from one into the other part (Sobczak \& Katrusiak, 2018). It was also shown that the compression of a crystal can be drastically changed in several different pressure-transmitting media (Cai et al., 2015).

When the temperature increases, the volume of most substances increases, too. Therefore, a rule of inverse effects of temperature and pressure was formulated (Hazen \& Finger, 1982). Naturally, the thermal expansion of volume is not necessarily positive, so the rule of inverse effects has exceptions. These exceptions are not only limited to the crystal volume, but they may also involve specific lattice parameters and structural dimensions. For example, if a crystal is most strongly compressed along direction $[c]$, the inverse rule would imply the strongest thermal expansion along this direction, too. This inverse relation is indeed observed in many crystals; however there are exceptions where the thermal expansion and compression are directly (as opposed to inversely) related. Such a positive relation can be explained by the secondary effects in the framework structure. The positive relation in the crystal $[\mathrm{Ag}($ ethylenediamine $)] \mathrm{NO}_{3}$ is due to the thermal vibrations of the $\mathrm{NO}_{3}$ anions in the cages of the wine-rack MOF (Cai \& Katrusiak, 2014). Analogous mechanisms can result in the positive relation of the temperature- and pressure-induced strain (Marciniak \& Katrusiak, 2017), or in drastically different anisotropy of strain on cooling and compression (Boldyreva et al., 1998).

Another feature often related to the systematic changes induced by temperature and pressure in crystals is their symmetry. It is important, because many properties of crystals are symmetry dependent. Therefore the prediction of possible symmetry changes is essential for choosing the optimum thermodynamic conditions for the synthesis of new phases of functional materials. Usually, the symmetry of crystals increases in the high-temperature phases. For example, ferroelectric or ferromagnetic phases exist at low temperature and above the critical temperature these materials become paraelectric and paramagnetic, respectively. At present, it appears that the inverse relation rule does not apply to the symmetry relations between low-pressure and high-pressure phases. There are similar numbers of low- and high-pressure phases that increase and lower their symmetry. The symmetry increase or decrease is straightforward for the symmetry group-subgroup relations (for example, for the prototypical ferroelectric $\mathrm{KH}_{2} \mathrm{PO}_{4}$, often abbreviated to KDP, the orthorhombic space group $F d d 2$ below $T_{\mathrm{c}}=122 \mathrm{~K}$ is the subgroup of the tetragonal $I \overline{4} 2 d$ above the $T_{\mathrm{c}}$ ). When no groupsubgroup relation exists for the transforming phases, their symmetry change can be assessed quantitatively by comparing the space-group order, and by comparing the $Z^{\prime}$ values (the number of symmetry-independent formula units). So far, there are numerous cases of both increased and decreased $Z^{\prime}$. The $Z^{\prime}$ increasing with pressure was observed, for example, in ethynylbenzene (Dziubek et al., 2007), pyrazine (Patyk et al., 2015), chlorpropamide (Seryotkin et al., 2013), thiourea (Tomkowiak \& Katrusiak, 2018), isopropyl alcohol (Ridout \& Probert, 2014) and urea when phase I transforms to phase III (Olejniczak et al., 2009; Roszak \& Katrusiak, 2017). In another group of compounds the $Z^{\prime}$ number decreases in the highpressure phase, for example in 6-chloro-4,4,5,7-tetramethyldihydrocoumarin (Rajewski et al., 2018), 3-hydroxy-4,5-dimethyl-1-phenylpyridazin-6-one (Roszak et al., 2016) and bromochlorofluoroacetic acid (Gajda et al., 2009). There are also structures where $Z^{\prime}$ initially increases and then decreases in consecutive phase transitions, for example in toluene $Z^{\prime}=1$, 2, 3 and 1 in phases I, II, III and IV, respectively (Marciniak $e t$ al., 2016), in urea phases I, III and IV $Z^{\prime}=0.25,1$ and 0.5 , in pyrimidine $Z^{\prime}=1,2,1$, in di-p-tolyl disulfide $Z^{\prime}$ changes from 1 in phase $\alpha$ to 2 in phase $\beta$ above $1.6 \mathrm{GPa}$, but the hidden phase $\gamma$ above $0.4 \mathrm{GPa}$ has $Z^{\prime}=1$ (Sobczak \& Katrusiak, 2017). The initial reduction and then increase of $Z^{\prime}$ from 2 in phase $\alpha$ to 0.5 in phase $\beta$ and to 1 in phase $\gamma$ was observed in 4,4'bipyridinium perchlorate (Anioła \& Katrusiak, 2017). There are also crystals where $Z^{\prime}$ increases at high temperature (from $1 / 12$ to 2 ) and in high-pressure (1/12 to 1/2) phases (Nowicki et al., 2012). It appears that there are two or more competing factors affecting the $Z^{\prime}$ number in high-pressure phases: (i) the energy of different intermolecular interactions of independent molecules with their environments in the crystal subjected to pressure increase, and this difference can be reduced by a molecular rearrangement to a lower $Z^{\prime}$; (ii) in some cases the space can be more tightly filled by molecules of different shape (for example conformers). There are also compounds, for example $N, N$-dimethylformamide (DMF), whose molecules strongly prefer to aggregate with increased $Z^{\prime}$ (Ratajczyk et al., 2019).

As already mentioned, for some substances preferentially forming pristine separate phases at ambient conditions, the high pressure changes this preference for multi-component crystals. There are numerous examples of multi-component crystals obtained at high pressure (Somayazulu et al., 1996; Loubeyre et al., 1993; Zieliński \& Katrusiak, 2015; Tomkowiak et al., 2013; Guńka et al., 2015; Anioła \& Katrusiak, 2017). It is not uncommon that several solvates of different composition can be obtained in narrow pressure ranges. For example, high pressure is required for obtaining the solvates of thiourea (Tomkowiak et al., 2013) and 4,4'-bipyridinium perchlorate (Anioła \& Katrusiak, 2017). No hydrates of these two compounds could be obtained above the pressure of $1.0 \mathrm{GPa}$, when water freezes into ice VI. Solvates of some pharma- 
ceutical compounds can be obtained exclusively at high pressure, paracetamol (Fabbiani et al., 2003) and L-alanine (Tumanov et al., 2010) serving as examples. Thus it appears that the main reason for the formation of high-pressure hydrates is the more dense packing possible for the structures composed from molecules of different sizes. However, when one of the mixture components crystallizes first (like water at $1.0 \mathrm{GPa}$ ), at still higher pressure the other component crystallizes separately, too (Tomkowiak et al., 2013; Zakharov et al., 2017).

The freezing point of the dissolved compound can be changed by its concentration in the solution. The arrangement of molecules in crystals often resembles the close packing of rigid spheres, with the coordination number of 12 or somewhat larger (Kitaigorodskii, 1973; Carugo et al., 2017). In this respect it resembles the closely packed rigid spheres, which fill $74 \%$ of the space, leaving the remaining quarter of space as empty voids. The high pressure naturally eliminates these voids, either by tighter packing of the compound itself or by relaxing the strain in the DAC chamber (in accordance with Le Châtelier's principle, as the whole DAC chamber is the closed system) by inducing the small liquid molecules into the voids. This was illustrated for the crystals of sphere-like molecules of compressed arsenolite, $\mathrm{As}_{4} \mathrm{O}_{6}$, and its helium clathrate $\mathrm{As}_{4} \mathrm{O}_{6} \cdot 2 \mathrm{He}$ (Guńka et al., 2015), methane compressed in hydrogen (Somayazulu et al., 1996), and for several salts of sphere-like 1,4-diazabicyclo[2.2.2]octane (usually abbreviated as DABCO) described by Olejniczak \& Katrusiak (2010, 2011).

It is reasonable to expect that the high-pressure polymorphs recovered to ambient conditions are denser than the polymorphs crystallized at ambient pressure. Their density difference is usually a few percent (Olejniczak et al., 2019; Boldyreva, 2008; Fabbiani et al., 2014). However, it is a surprising effect that after a solvate molecule is added to the structure, the molecular volume $\left(V_{\mathrm{m}}\right)$ of such a larger formula unit is smaller than the original $V_{\mathrm{m}}$ of the pristine compound. It was found that high pressure is capable of producing such a solvate, 5,6-dimethylbenzimidazole hemihydrate, whose volume after the recovery to ambient pressure is smaller than that of the pristine compound and this hemihydrate could be stored in an open vial for months. Moreover, the hemihydrate acquired good solubility in water, unlike the anhydrate (Zieliński \& Katrusiak, 2015). We are aware of only one other compound, a MOF with its pores filled, which expands after removing the guests (Andrzejewski et al., 2017).

Structural effects induced by high pressure in crystals can be so strong that they cannot be regarded solely as a physical transformation, as they change the chemical character of substances, even before the chemical reaction actually takes place. For example, the bond lengths, molecular conformation, coordination and interactions can be reversibly changed and correlated with the properties of materials under study. Thus, to some extent, high pressure can substitute chemical reactions and considerably facilitate the research on functional materials, in the search for their optimum composition. For example, high-pressure experiments on a promising photovoltaic material, methylammonium lead triiodide, $\mathrm{MAPbI}_{3}$, where MA stands for $\left[\mathrm{CH}_{3} \mathrm{NH}_{3}\right]^{+}$, reveal the correlation between the structural features, such as the $\mathrm{Pb}-\mathrm{I}$ bond distances and $\mathrm{Pb}-$ $\mathrm{I}-\mathrm{Pb}$ angles, with the optical absorption and energy band gap (Szafrański \& Katrusiak, 2016, 2017; Onoda-Yamamuro et al., 1992; Liu et al., 2017). Similar high-pressure experiments were applied for investigating the structure-property relations of other photovoltaic perovskites (Wang, Wang \& Zou, 2016; Wang, Wang, Xiao et al., 2016).

High pressure can drastically change the types of interactions in crystals. In accordance with Le Châtelier's principle, the weakest interactions are most affected. In crystals of different types, the hierarchy of pressure-induced transformations can be predicted depending on the associated energy changes. Depending on the pressure range and compressed material, different types of transformations can be specified (Boehler, 2005; Prewitt \& Downs, 1998; Grochala et al., 2007). Flexible molecules are likely to transform their conformation (Bujak $e t$ al., 2008; Anioła \& Katrusiak, 2017). Another type of possible pressure-induced change is neutral-ionic transitions (Macchi et al., 2010; Cointe et al., 1996; Luty, 1987, 1992; Luty \& Kuchta, 1987; Sikora \& Katrusiak, 2013). They illustrate the effects of increased energy of interactions at high pressure on the electronic structure of molecules. Neutral-ionic transitions have been observed for typical charge-transfer complexes, but also for the $\mathrm{OH}$... O hydrogen-bonded oxalic acid dihydrate, between different molecules (Macchi et al., 2010), and for $\mathrm{NH} \cdots \mathrm{N}$-bonded pyrazole the favoured positions of protons between independent molecules are eliminated at $0.45 \mathrm{GPa}$ and all molecules become neutral in phase $\beta$ (Sikora \& Katrusiak, 2013).

In most cases flexible molecules in crystals assume the energetically favoured conformation, so it is often assumed that the crystallographic structure is an indication of the conformational preferences. Naturally, there are notable exceptions, such as biphenyl. As the 'soft' parameters are easily modified, the pressure-induced transformations usually increase the energy of conformers. Exceptions to this rule have also been reported (Cai \& Katrusiak, 2013).

Sufficiently high pressure destabilizes unsaturated compounds. It was assessed 35 years ago that unsaturated compounds polymerize at about $10 \mathrm{GPa}$ (Nicol \& Yin, 1984). For example, at $300 \mathrm{~K}$ carbon disulfide $\mathrm{CS}_{2}$ polymerizes at $8 \mathrm{GPa}$ (Dziubek \& Katrusiak, 2004), benzene at $24 \mathrm{GPa}$ (Budzianowski \& Katrusiak, 2006; Wen et al., 2011), benzene:perfluorobenzene mixture at $25 \mathrm{GPa}$ (Wang, Wang, Zheng et al., 2016) but acetonitrile trimerizes at $1.6 \mathrm{GPa}$ above $400 \mathrm{~K}$ (Olejniczak \& Katrusiak, 2008). Pressure-induced polymerization is currently considered as a method for obtaining super-hard and chemically inert materials. Some of these materials display special properties, like electric conductivity.

Also, rules of the behaviour of chiral compounds at high pressure can be formulated. For example, all pressure-induced first-order phase transitions of any racemate act towards increasing the density racemate $_{\text {density }}$ enantiomer ratio. The phase transition of DL-maleic acid at $0.7 \mathrm{GPa}$ changes the density 
ratio from 0.960 to 1.007 . Consequently, Wallach's rule, disobeyed for mandelic acid at normal conditions, is restored. Likewise, all pressure-induced first-order phase transitions in enantiomers reduce the density racemate $_{\text {density }}$ enantiomer ratio (Marciniak et al., 2014).

The consideration of the atomic/ionic volumes occupied in the structure leads to other systematic changes in the compressed compounds. The cations, usually smaller, with more compact electronic orbitals, are 'harder to compress' than anions. Consequently, in most coordination compounds where the anions and electronegative atoms coordinate a central cation, high pressure usually increases the coordination number (Półrolniczak et al., 2018). It was found that the temperature effect is the opposite, in accordance with the rule of inverse effects of pressure and temperature (Hazen \& Finger, 1982), introduced above. It can be noted that the increased coordination was pointed out for silicates too (Liebau, 1984; Sowa, 1988), which may be an indication that similar rules apply to oxides. However, the revealed reduction of the oxidation of iron at very high pressure (Lavina et al., 2011) contrasts with such a general statement.

Last, but not least, the high-pressure research of recent years demonstrates clearly that the properties of crystals may strongly depend on their environment. In many crystals the pressure-induced transformations depend not only on the structure, but also on the pressure-transmitting medium (Sobczak \& Katrusiak, 2018, 2019; Cai et al., 2015; Zakharov, Seryotkin et al., 2016). The interactions of the crystal surface with the environment have significant implications. Consequently, some of the presently accepted results may require verification. For example, high-pressure and low-temperature phases can be metastable, and when compressed in good solvents they would recrystallize in new phases or in new solvates more stable at these conditions. The transformation into new polymorphs may require that the sample be dissolved (Paliwoda et al., 2012; Sobczak \& Katrusiak, 2017). Alternatively, the transport of the surrounding medium into the bulk of the crystal, without a drastic reorganization of its structure, can be induced by pressure, even when no pores are present. Finally, quick and slow compression in a highly viscous medium may result in different transformations, either monotonic or anomalous ones. The anomalous effects are particularly strong for soft framework materials (Moggach et al., 2009; Ogborn et al., 2012; Graham et al., 2011), some of which undergo reversible or irreversible amorphization (Bennett et al., 2011; Bennett \& Cheetham, 2014).

\section{Conclusions}

Owing to the progress in DAC designs and in the auxiliary equipment of DACs, a steadily increasing amount of highpressure results is being published in scientific journals. It can be envisaged that the high-pressure results will continue to flow and will contribute to a more complete understanding of the fundamental and applied sciences. It appears that all the broadly understood material sciences can benefit from highpressure experiments. Presently, high pressure is used in many different research areas. One of the most common directions is the investigation of intermolecular interactions, generation of new polymorphs (including pressure-induced amorphous phases), solvates and phase transitions. Particularly inspiring are pharmaceutical applications (Neumann et al., 2015; Fabbiani et al., 2014; Zakharov, Goryainov et al., 2016; Ridout et al., 2014; Lee et al., 2014), and new directions of highpressure applications in the field of organic compounds and molecular crystals (Andrzejewski \& Katrusiak, 2017a,b; Hejny $\&$ Minkov, 2015). New information has been gained from highpressure research in structural biology (Fourme et al., 2004) and microbiology (Meersman et al., 2013; Meersman \& McMillan, 2014). In the coming years the effects of pressure on the crystal surface will certainly be studied more, which will find applications in sensing devices (Runowski et al., 2019). Another domain of emerging research is processes under dynamically changing pressure (Evans et al., 2007). Undoubtedly, during the last six decades the DAC has wide opened the door to unexplored high-pressure territories. Although the sample is confined to a small space between two diamond tips, the DAC supported by numerous auxiliary experimental techniques has markedly expanded the capabilities of high-pressure research. Will any of the further developments in high-pressure research surpass the impact of the DAC? Presently, the range of dynamical high-pressure research is further extended by laser shock experiments on pre-compressed samples (Wang, Coppari et al., 2016).

\section{Nomenclature comments}

The terms 'low' or 'high' pressure are usually related to the ambient atmosphere at sea level (0.1 MPa). In crystallography the term 'high pressure' is generally applied for describing pressures many times higher, which are capable of significantly compressing liquids and crystals. The European High Pressure Research Group (EHPRG) in its Governing Rules and Notes (EHPRG Governing Rules \& Notes, 2015) states: 'High pressure is here defined to mean above 1000 bar or $100 \mathrm{MPa}$ '. This pressure value is close to that at the deepest point of the Mariana Trench, about $11 \mathrm{~km}$ below sea level. Incidentally, the DAC as well as the ruby calibration are best suited for pressure higher than $0.1 \mathrm{GPa}$. However, pressure considerably lower than $0.1 \mathrm{GPa}$ can also significantly affect molecular systems. It can be observed in the location of the triple point in very many $p-T$ phase diagrams; there are also examples where a fraction of $1 \mathrm{GPa}$ changes the crystallization preferences of polymorphs or multiple-component crystals. The adsorption of gases in porous materials, such as MOFs, often changes drastically between 0.1 and 5.0 $\mathrm{MPa}$.

The hydrostatic conditions at room temperature can exist up to $11.3 \mathrm{GPa}$, when helium freezes, which is the substance hardest to freeze by pressure known so far. Above $11.3 \mathrm{GPa}$ all conditions can be described either as pseudo-hydrostatic (when the sample is considerably harder than the surrounding pressure-transmitting medium) or as non-hydrostatic. In many experiments special care is taken to secure the possibly hydrostatic conditions which are desirable for accurately 
describing the physical or chemical phenomena. For example, sample annealing can reduce the non-hydrostatic strain. On the other hand, samples are often compressed in a DAC intentionally without any hydrostatic fluid at all, for example in order to maximize the Raman signal. In some cases, solid pseudo-hydrostatic media much harder than the compounds under study are applied, which introduces considerable discrepancies, particularly in anisotropic systems.

\section{Funding information}

This study was partly supported by project Opus 10 UMO2015/19/B/ST5/00262 from the Polish National Science Center.

\section{References}

Allan, D. R. \& Clark, S. J. (1999). Phys. Rev. Lett. 82, 34643467.

Allan, D. R., Clark, S. J., Brugmans, M. J. P., Ackland, G. J. \& Vos, W. L. (1998). Phys. Rev. B, 58, R11809-R11812.

Andrzejewski, M., Casati, N. \& Katrusiak, A. (2017). Dalton Trans. 46, 14795-14803.

Andrzejewski, M. \& Katrusiak, A. (2017a). J. Phys. Chem. Lett. 8, 279-284.

Andrzejewski, M. \& Katrusiak, A. (2017b). J. Phys. Chem. Lett. 8, 929-935.

Anioła, M. \& Katrusiak, A. (2017). Cryst. Growth Des. 17, 31343141.

Barrio, M., Huguet, J., Rietveld, I. B., Robert, B., Céolin, R. \& Tamarit, J. L. (2017). J. Pharm. Sci. 106, 1538-1544.

Barrio, M., Maccaroni, E., Rietveld, I. B., Malpezzi, L., Masciocchi, N., Céolin, R. \& Tamarit, J. L. (2012). J. Pharm. Sci. 101, 10731078.

Bennett, T. D. \& Cheetham, A. K. (2014). Acc. Chem. Res. 47, 15551562.

Bennett, T. D., Simoncic, P., Moggach, S. A., Gozzo, F., Macchi, P., Keen, D. A., Tan, J.-C. \& Cheetham, A. K. (2011). Chem. Commun. 47, 7983-7985.

Bertani, R., Mali, M., Roos, J. \& Brinkmann, D. (1992). Rev. Sci. Instrum. 63, 3303-3306.

Bezzu, C. G., Burt, L. A., McMonagle, C. J., Moggach, S. A., Kariuki, B. M., Allan, D. R., Warren, M. \& McKeown, N. B. (2019). Nat. Mater. 18, 740-745.

Boehler, R. (2005). Mater. Today, 8, 34-42.

Boldyreva, E. V. (2008). Acta Cryst. A64, 218-231.

Boldyreva, E. V., Naumov, D. Y. \& Ahsbahs, H. (1998). Acta Cryst. B54, 798-808.

Budzianowski, A. \& Katrusiak, A. (2006). Acta Cryst. B62, 94101.

Bujak, M., Podsiadło, M. \& Katrusiak, A. (2008). Chem. Commun. pp. 4439-4441.

Cai, W., Gładysiak, A., Anioła, M., Smith, V. J., Barbour, L. J. \& Katrusiak, A. (2015). J. Am. Chem. Soc. 137, 9296-9301.

Cai, W. \& Katrusiak, A. (2013). J. Phys. Chem. C, 117, 2146021465 .

Cai, W. \& Katrusiak, A. (2014). Nat. Commun. 5, 4337.

Carugo, O., Blatova, O. A., Medrish, E. O., Blatov, V. A. \& Proserpio, D. M. (2017). Sci. Rep. 7, 13209.

Coasne, B., Haines, J., Levelut, C., Cambon, O., Santoro, M., Gorelli, F. \& Garbarino, G. (2011). Phys. Chem. Chem. Phys. 13, 2009620099.

Coudert, F.-X. (2015). Acta Cryst. B71, 585-586.

Dubrovinskaia, N., Dubrovinsky, L., Solopova, N. A., Abakumov, A., Turner, S., Hanfland, M., Bykova, E., Bykov, M., Prescher, C., Prakapenka, V. B., Petitgirard, S., Chuvashova, I., Gasharova, B., Mathis, Y.-L., Ershov, P., Snigireva, I. \& Snigirev, A. (2016). Sci. Adv. 2, e1600341.
Dziubek, K., Podsiadło, M. \& Katrusiak, A. (2007). J. Am. Chem. Soc. 129, 12620-12621.

Dziubek, K. F. \& Katrusiak, A. (2004). J. Phys. Chem. B, 108, 19089 19092.

EHPRG Governing Rules \& Notes (2015). Issued 10.8.2015, Section 3.1, Point 2, http://www.ehprg.org/docs/2015_EHPRG_Governing_Rules.pdf; downloaded 30.12.2018.

Evans, W. J., Yoo, C.-S., Lee, G. W., Cynn, H., Lipp, M. J. \& Visbeck, K. (2007). Rev. Sci. Instrum. 78, 073904.

Fabbiani, F. P. A., Buth, G., Levendis, D. C. \& Cruz-Cabeza, A. J. (2014). Chem. Commun. 50, 1817-1819.

Fabbiani, F. P., Allan, D. R., Dawson, A., David, W. I., McGregor, P. A., Oswald, I. D., Parsons, S. \& Pulham, C. R. (2003). Chem. Commun. pp. 3004-3005.

Fabbiani, F. P. A. \& Pulham, C. R. (2006). Chem. Soc. Rev. 35, 932 942.

Fourme, R. (1968). J. Appl. Cryst. 1, 23-30.

Fourme, R., Girard, E., Kahn, R., Aseone, I., Mezouar, M., Lin, T. \& Johnson, J. E. (2004). High-Pressure Crystallography. NATO Science Series (Series II: Mathematics, Physics and Chemistry), Vol. 140, edited by A. Katrusiak \& P. McMillan, pp. 527-542. Dordrecht: Springer.

Gajda, R., Katrusiak, A. \& Crassous, J. (2009). CrystEngComm, 11, 2668-2676.

Goncharenko, N. (2004). High-Pressure Crystallography, edited by A. Katrusiak \& P. F. McMillan, pp. 321-340. Dordrecht: Kluwer.

Graf, D. E., Stillwell, R. L., Purcell, K. M. \& Tozer, S. W. (2011). High Pressure Res. 31, 533-543.

Graham, A. J., Allan, D. R., Muszkiewicz, A., Morrison, C. A. \& Moggach, S. A. (2011). Angew. Chem. Int. Ed. 50, 1113811141.

Grochala, W., Hoffmann, R., Feng, J. \& Ashcroft, N. W. (2007). Angew. Chem. Int. Ed. 46, 3620-3642.

Groom, C. R., Bruno, I. J., Lightfoot, M. P. \& Ward, S. C. (2016). Acta Cryst. B72, 171-179.

Guńka, P. A., Dziubek, K. F., Gładysiak, A., Dranka, M., Piechota, J., Hanfland, M., Katrusiak, A. \& Zachara, J. (2015). Cryst. Growth Des. 15, 3740-3745.

Hazen, R. M. \& Finger, L. (1982). Comparative Crystal Chemistry. New York: John Wiley \& Sons.

Hejny, C. \& Minkov, V. S. (2015). IUCrJ, 2, 218-229.

Huppertz, H. (2011). Chem. Commun. 47, 131-140.

Jackson, D. D., Aracne-Ruddle, C., Malba, V., Weir, S. T., Catledge, S. A. \& Vohra, Y. K. (2003). Rev. Sci. Instrum. 74, 2467-2471.

Jamieson, J. C., Lawson, A. W. \& Nachtrieb, N. D. (1959). Rev. Sci. Instrum. 30, 1016-1019.

Jayaraman, A. (1983). Rev. Mod. Phys. 55, 65-108.

Katrusiak, A. (2008). Acta Cryst. A64, 135-148.

Katrusiak, A. (2018). International Tables for Crystallography, Vol. H, ch. 2.7, pp. 156-173. Chester: International Union of Crystallography.

Katrusiak, A., Szafrański, M. \& Podsiadło, M. (2011). Chem. Commun. 47, 2107-2109.

Kitaigorodskii, A. I. (1973). Molecular Crystals and Molecules. London: Academic Press.

Lavina, B., Dera, P., Kim, E., Meng, Y., Downs, R. T., Weck, P. F., Sutton, S. R. \& Zhao, Y. (2011). Proc. Natl Acad. Sci. USA, 108, 17281-17285.

Le Cointe, M., Lemée-Cailleau, M. H., Cailleau, H. \& Toudic, B. (1996). J. Mol. Struct. 374, 147-153.

Lee, R., Howard, J. A. K., Probert, M. R. \& Steed, J. W. (2014). Chem. Soc. Rev. 43, 4300-4311.

Lee, Y., Vogt, T., Hriljac, J. A., Parise, J. B. \& Artioli, G. (2002). J. Am. Chem. Soc. 124, 5466-5475.

Liebau, F. (1984). Inorg. Chim. Acta, 89, 1-7.

Liu, G., Kong, L., Gong, J., Yang, W., Mao, H., Hu, Q., Liu, Z., Schaller, R. D., Zhang, D. \& Xu, T. (2017). Adv. Funct. Mater. 27, 1604208. 
Loubeyre, P., Jean-Louis, M., LeToullec, R. \& Charon-Gérard, L. (1993). Phys. Rev. Lett. 70, 178-181.

Luty, T. (1987). J. Chem. Phys. 87, 3137-3142.

Luty, T. (1992). J. Phys. Soc. Jpn, 61, 3636-3642.

Luty, T. \& Kuchta, B. (1987). Phys. Rev. B, 35, 8542-8550.

Macchi, P., Casati, N., Marshall, W. G. \& Sironi, A. (2010). CrystEngComm, 12, 2596-2603.

Mao, H., Shu, J., Shen, G., Hemley, R. J., Li, B. \& Singh, A. K. (1998). Nature, 396, 741-743.

Marciniak, J., Andrzejewski, M., Cai, W. \& Katrusiak, A. (2014). J. Phys. Chem. C, 118, 4309-4313.

Marciniak, J., Bąkowicz, J., Dobrowolski, M. A., Dziubek, K. F., Kaźmierczak, M., Paliwoda, D., Rajewski, K. W., Sobczak, S., Stachowicz, M. \& Katrusiak, A. (2016). Cryst. Growth Des. 16, 1435-1441.

Marciniak, J. \& Katrusiak, A. (2017). J. Phys. Chem. C, 121, 2230322309.

Marizy, A., Guigue, B., Occelli, F., Leridon, B. \& Loubeyre, P. (2017). High Pressure Res. 37, 465-474.

McKellar, S. C. \& Moggach, S. A. (2015). Acta Cryst. B71, 587607.

Meersman, F., Daniel, I., Bartlett, D. H., Winter, R., Hazael, R. \& McMillan, P. F. (2013). Rev. Mineral. Geochem. 75, 607-648.

Meersman, F. \& McMillan, P. F. (2014). Chem. Commun. 50, 766775.

Meyer, B., Barthel, S., Mace, A., Vannay, L., Guillot, B., Smit, B. \& Corminboeuf, C. (2019). J. Phys. Chem. Lett. 10, 1482-1488.

Moggach, S. A., Bennett, T. D. \& Cheetham, A. K. (2009). Angew. Chem. 121, 7221-7223.

Moggach, S. A., Parsons, S. \& Wood, P. A. (2008). Crystallogr. Rev. 14, 143-184.

Neumair, S. C., Kaindl, R. \& Huppertz, H. (2010). Z. Naturforsch. B, 65, 1311-1317.

Neumann, M. A., van de Streek, J., Fabbiani, F. P. A., Hidber, P. \& Grassmann, O. (2015). Nat. Commun. 6, 7793.

Nicol, M. \& Yin, G. Z. (1984). J. Phys. Colloq. 45, 163-172.

Nowicki, W., Olejniczak, A., Andrzejewski, M. \& Katrusiak, A. (2012). CrystEngComm, 14, 6428-6434.

Oganov, A. R. (2018). Faraday Discuss. 211, 643-660.

Oganov, A. R., Lyakhov, A. O. \& Valle, M. (2011). Acc. Chem. Res. 44, 227-237.

Ogborn, J. M., Collings, I. E., Moggach, S. A., Thompson, A. L. \& Goodwin, A. L. (2012). Chem. Sci. 3, 3011-3017.

Olejniczak, A. \& Katrusiak, A. (2008). J. Phys. Chem. B, 112, $7183-$ 7190.

Olejniczak, A. \& Katrusiak, A. (2010). CrystEngComm, 12, 25282532.

Olejniczak, A. \& Katrusiak, A. (2011). Cryst. Growth Des. 11, 2250 2256.

Olejniczak, A., Katrusiak, A., Podsiadło, M. \& Katrusiak, A. (2019). Cryst. Growth Des. 19, 1832-1838.

Olejniczak, A., Ostrowska, K. \& Katrusiak, A. (2009). J. Phys. Chem. $C, \mathbf{1 1 3}, 15761-15767$.

Onoda-Yamamuro, N., Yamamuro, O., Matsuo, T. \& Suga, H. (1992). J. Phys. Chem. Solids, 53, 277-281.

Paliwoda, D., Dziubek, K. F. \& Katrusiak, A. (2012). Cryst. Growth Des. 12, 4302-4305.

Patyk, E., Podsiadło, M. \& Katrusiak, A. (2015). Cryst. Growth Des. 15, 5670-5674.

Piermarini, G. J., Mighell, A. D., Weir, C. E. \& Block, S. (1969). Science, 165, 1250-1255.

Pinkowicz, D., Rams, M., Mišek, M., Kamenev, K. V., Tomkowiak, H., Katrusiak, A. \& Sieklucka, B. (2015). J. Am. Chem. Soc. 137, 87958802.

Podsiadło, M., Olejniczak, A. \& Katrusiak, A. (2017). Cryst. Growth Des. 17, 228-232.

Półrolniczak, A., Sobczak, S. \& Katrusiak, A. (2018). Inorg. Chem. 57, 8942-8950.
Prewitt, C. T. \& Downs, R. T. (1998). In Ultrahigh Pressure Mineralogy: Physics and Chemistry of the Earth's Deep Interior, edited by R. J. Hemley, pp. 283-318. Walter de Gruyter GmbH.

Price, S. L. (2018). Proc. R. Soc. A, 474, 20180351.

Rajewski, K. W., Bukalska, I. \& Katrusiak, A. (2018). Cryst. Growth Des. 18, 3187-3192.

Ratajczyk, P., Sobczak, S. \& Katrusiak, A. (2019). Cryst. Growth Des. 19, 896-901.

Ridout, J., Price, L. S. Howard, J. A. K. \& Probert, M. (2014). Cryst. Growth Des. 14, 3384-3391.

Ridout, J. \& Probert, M. (2014). CrystEngComm, 16, 7397-7400.

Roszak, K. \& Katrusiak, A. (2017). J. Phys. Chem. C, 121, 778784.

Roszak, K., Katrusiak, A. \& Katrusiak, A. (2016). Cryst. Growth Des. 16, 3947-3953.

Runowski, M., Sobczak, S., Marciniak, J., Bukalska, I., Lis, S. \& Katrusiak, A. (2019). Nanoscale, 11, 8718-8726.

Sakai, T., Yagi, T., Irifune, T., Kadobayashi, H., Hirao, N., Kunimoto, T., Ohfuji, H., Kawaguchi-Imada, S., Ohishi, Y., Tateno, Sh. \& Hirose, K. (2018). High Pressure Res. 38, 107-119.

Seryotkin, Y. V., Drebushchak, T. N. \& Boldyreva, E. V. (2013). Acta Cryst. B69, 77-85.

Sikora, M. \& Katrusiak, A. (2013). J. Phys. Chem. C, 117, 1066110668.

Smith, R. F., Eggert, J. H., Jeanloz, R., Duffy, T. S., Braun, D. G., Patterson, J. R., Rudd, R. E., Biener, J., Lazicki, A. E., Hamza, A. V., Wang, J., Braun, T., Benedict, L. X., Celliers, P. M. \& Collins, G. W. (2014). Nature, 511, 330-333.

Sobczak, S., Drożdż, W., Lampronti, G. I., Belenguer, A. M., Katrusiak, A. \& Stefankiewicz, A. R. (2018). Chem. Eur. J. 24, 8769-8773.

Sobczak, S. \& Katrusiak, A. (2017). J. Phys. Chem. C, 121, 2539 2545.

Sobczak, S. \& Katrusiak, A. (2018). Cryst. Growth Des. 18, 1082 1089.

Sobczak, S. \& Katrusiak, A. (2019). Inorg. Chem. 58, 1177311781.

Somayazulu, M. S., Finger, L. W., Hemley, R. J. \& Mao, H. K. (1996). Science, 271, 1400-1402.

Sowa, H. (1988). Z. Kristallogr. 184, 257-268.

Szafrański, M. \& Katrusiak, A. (2016). J. Phys. Chem. Lett. 7, 34583466.

Szafrański, M. \& Katrusiak, A. (2017). J. Phys. Chem. Lett. 8, 24962506.

Tomkowiak, H. \& Katrusiak, A. (2018). J. Phys. Chem. C, 122, 50645070.

Tomkowiak, H., Olejniczak, A. \& Katrusiak, A. (2013). Cryst. Growth Des. 13, 121-125.

Toscani, S., Céolin, R., Minassian, L. T., Barrio, M., Veglio, N., Tamarit, J. L., Louër, D. \& Rietveld, I. B. (2016). Int. J. Pharm. 497, 96-105.

Tumanov, N. A., Boldyreva, E. V., Kolesov, B. A., Kurnosov, A. V. \& Quesada Cabrera, R. (2010). Acta Cryst. B66, 458-471.

Van Valkenburg, A. (1962). Rev. Sci. Instrum. 33, 1462.

Wang, J., Coppari, F., Smith, R. F., Eggert, J. H., Lazicki, A. E., Fratanduono, D. E., Rygg, J. R., Boehly, T. R., Collins, G. W. \& Duffy, T. S. (2016). Phys. Rev. B, 94, 104102.

Wang, L., Wang, K., Xiao, G., Zeng, Q. \& Zou, B. (2016). J. Phys. Chem. Lett. 7, 5273-5279.

Wang, L., Wang, K. \& Zou, B. (2016). J. Phys. Chem. Lett. 7, 25562562.

Wang, Y., Wang, L., Zheng, H., Li, K., Andrzejewski, M., Hattori, T., Sano-Furukawa, A., Katrusiak, A., Meng, Y., Liao, F., Hong, F. \& Mao, H. (2016). J. Phys. Chem. C, 120, 29510-29519.

Weir, C. E., Lippincott, E. R., Van Valkenburg, A. \& Bunting, N. E. (1959). J. Res. Natl Bur. Stand. Sect. A, 63, 55-62.

Wen, X.-D., Hoffmann, R. \& Ashcroft, N. W. (2011). J. Am. Chem. Soc. 133, 9023-9035. 
Zakharov, B. A., Goryainov, S. V. \& Boldyreva, E. V. (2016). CrystEngComm, 18, 5423-5428.

Zakharov, B. A., Gribov, P. A., Matvienko, A. A. \& Boldyreva, E. V. (2017). Z. Kristallogr. 232, 751-757.

Zakharov, B. A., Marchuk, A. S. \& Boldyreva, E. V. (2015). CrystEngComm, 17, 8812-8816.
Zakharov, B. A., Seryotkin, Y. V., Tumanov, N. A., Paliwoda, D., Hanfland, M., Kurnosov, A. V. \& Boldyreva, E. V. (2016). RSC Adv. 6, 92629-92637.

Zieliński, W. \& Katrusiak, A. (2015). CrystEngComm, 17, 54685473.

Zurek, E. \& Bi, T. (2019). J. Chem. Phys. 150, 050901. 